\title{
Predicting Ruthenium Catalysed Hydrogenation of Esters using Ma- chine Learning
}

\author{
Challenger Mishra, ${ }^{* a}$ Niklas von Wolff, ${ }^{* b}$ Abhinav Tripathi, ${ }^{\mathrm{c}}$ Eric Brémond, ${ }^{\mathrm{d}}$ Annika Preiss, ${ }^{\mathrm{c}}$ Amit Ku- \\ mar*c
}

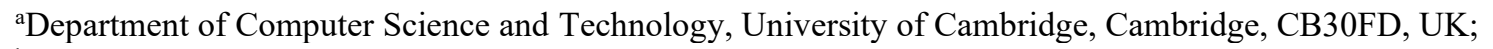

bLaboratoire d'Electrochimie Moléculaire, Université de Paris, CNRS, F-75006 Paris, France;

'School of Chemistry, University of St. Andrews, St. Andrews, KY169ST, UK;

dITODYS, Université de Paris, CNRS, F-75006 Paris, France.

\begin{abstract}
Catalytic hydrogenation of esters is a sustainable approach for the production of fine chemicals, and pharmaceutical drugs. However, the efficiency and cost of catalysts are often the bottlenecks in the commercialization of such technologies. The conventional approach of catalyst discovery is based on empiricism that makes the discovery process time-consuming and expensive. There is an urgent need to develop effective approaches to discover efficient catalysts for hydrogenation reactions. We demonstrate here the approach of machine learning for the prediction of outcomes for the catalytic hydrogenation of esters. Our models can predict the reaction yields with high mean accuracies of up to $91 \%$ (test set) and suggest that the use of certain chemical descriptors selectively can result in a more accurate model. Furthermore, catalysts and some of their corresponding descriptors can also be predicted with mean accuracies of $85 \%$, and $>90 \%$, respectively.
\end{abstract}

The catalytic hydrogenation of esters to alcohols is an atomeconomic and sustainable approach in organic synthesis with significant applications in the production of various fine chemicals such as detergents, cosmetics, flavors, fragrances, and pharmaceutical drugs. ${ }^{1}$ The concept has also been expanded to the hydrogenation of polyesters to enable a circular economy. ${ }^{2}$ In the past, several homogeneous and heterogeneous catalysts have been developed, among which well-defined ruthenium complexes represent the state-of-the-art catalysts for the hydrogenation of esters to alcohols. ${ }^{1,3}$ However, most of such catalysts exhibit low TONs (e.g. < 200), and operate under harsh conditions (e.g. temperature $>100{ }^{\circ} \mathrm{C}$, and pressure $>20$ bars) making this approach expensive and incompatible for molecules containing other sensitive or reducible functional groups. Thus, the true utilization of hydrogenation methodology relies on finding an optimum catalyst that can hydrogenate an ester with high activity and selectivity and under mild conditions (e.g., room temperature, and ambient pressure). Our current conventional approach of catalysis development partly fails to achieve this due to its multiple limitations such as (a) empirical screening of several parameters such as solvent, temperature, pressure, time, additive, etc. can only be limited to certain extent; (b) syntheses of well-defined ruthenium catalysts often involve complex multi-step processes limiting the scope of complexes that can be studied, (c) lack of mechanistic understanding of new complexes limits its application in catalysis, and (d) intrinsic limitation of the human brain to find a pattern in large data collection restricts us to a smaller dataset. Chemoinformatics provides an attractive alternative to the conventional empirical approach. Although a mechanistic understanding of the underlying class of reactions can be highly beneficial in such a venture, one can often find patterns in large datasets of chemical reactions even without this. This can be accomplished by deploying novel machine-learning methodologies and architectures tailored to such applications. ${ }^{4}$ This can facilitate the discovery of desirable catalyst designs and catalytic conditions, bypassing the complexity of empirical synthesis and screening.

Although powerful, the application of the tool of machine learning (ML) for the discovery of molecular catalysts is in its nascent phase and growing. A few reports have been published on the development of predictive models for catalytic reactions using various machine learning architectures dictated by the nature of the reaction, molecular catalyst, and available dataset. For example, Kozlovski utilized Quantitative Structure Selectivity Relationship (QSSR) models for the prediction of the catalytic alkylation of aldehydes using beta-amino alcohol catalysts. ${ }^{5}$ Sigman and co-workers have developed predictive models for several asymmetric catalytic reactions using multivariate regression models. ${ }^{6-15}$ Along this direction, Doyle, and coworkers have used a random forest model to predict the yield of catalytic C-N cross-coupling reactions. ${ }^{16}$ Denmark has recently reported a computationally guided workflow and a highly accurate predictive model for chiral phosphoric-acid catalysed thiol addition to $\mathrm{N}$-acylimines using deep feed-forward neural networks. ${ }^{17}$ A predictive model for the asymmetric hydrogenation of alkenes and imines catalysed by chiral binapthyl catalysts has been recently reported by Sunoj where a root-mean-square error (rmse) of about $8.4 \pm 1.8 \%$ (enantiomeric excess) was obtained using a random forest model. ${ }^{18}$

Considering the contemporary interests in developing sustainable catalysts for the hydrogenation of esters, we report here the application of ML to predict the outcome of ester hydrogenation using well-defined ruthenium catalysts. Our approach involves the following three steps: (1) dataset construction, and exploratory data analysis, (2) creation of chemical descriptors for catalysts, and esters, and (3) development of predictive 
models using ML frameworks such as neural networks (NNs), and Gaussian processes (GPs).

1. Dataset construction: We created a dataset of reactions involving hydrogenation of esters by well-defined ruthenium complexes from existing (peer-reviewed) literature. This choice is guided by two factors. Firstly, homogeneous ruthenium catalysts are known for their high activity towards hydrogenation of esters making this family a suitable choice for potential superior catalysts. Secondly, substantial research outputs in the past on several types of ruthenium catalysts present systematic data needed for ML studies. Only those examples have been included in the dataset where the structure of ruthenium catalysts are well-defined, and they operate under neutral or basic conditions. Mechanistically, all the catalysts (with a few exceptions) have been proposed to operate via non-redox metal-ligand cooperation. Thus, our dataset consists of 460 hydrogenation reactions involving 85 ruthenium catalysts and 114 esters (Figure 1). Each reaction or datapoint is characterized by 12 parameters - catalyst structure, ester structure, amount of ester ( $\mathrm{mmol})$, catalyst loading (mol\%), base structure, base loading (mol\%), temperature $\left({ }^{\circ} \mathrm{C}\right)$, pressure of $\mathrm{H}_{2}$ (bar), reaction time (h), solvent structure, solvent amount $(\mathrm{mL})$, and yield $(\%)$.

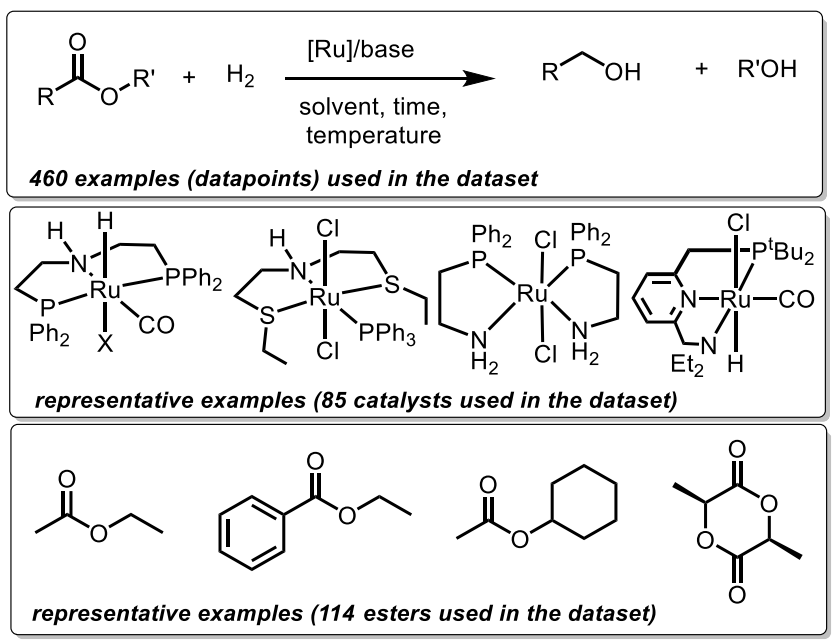

Figure 1. Representative structures of catalysts, and esters used in our dataset.

\section{Creation of chemical descriptors:}

In order to successfully use the dataset to develop a generalizable ML model, it is important to transform the structures and properties of catalysts, esters, bases, and solvents to informative numbers called chemical descriptors as also recently highlighted by Grzybowski and co-workers. ${ }^{19}$ Significant work has been done on the development of various types of chemical descriptors and their importance for the development of ML models for catalytic reactions. ${ }^{20-23}$ Aspuru-Guzik and Balcells have used graph or connectivity-based chemical descriptors computed using autocorrelation function from DFT optimized structures for a transition-metal complex. ${ }^{24}$ Choice of descriptors and their calculations using DFT, experiments, or ML can also depend on the size of the dataset. In general, there is however a tradeoff between descriptor accuracy and computational cost. In the case of large datasets, graph-or connectivity-based descriptors can be used successfully in ML approaches with minimum computational cost. ${ }^{24}$ In this work, the design of suitable descriptors is challenging due to the small size of the dataset, the variety of ligand architectures and complex geometries, the known importance of solvent interactions in the studied catalysts operating through metal-ligand cooperation, ${ }^{25-28}$ as well as unknown mechanisms and involvement of several rate-limiting states. ${ }^{29-31}$ We used a combination of DFT-based as well as experimentally estimated descriptors in addition to graph-based descriptors (Figure 2). Catalysts have been represented by three types of chemical descriptors. The first type is the graph-based descriptor calculated using an autocorrelation function of depth 4. ${ }^{24}$ The second type is sterics-based descriptors calculated using topographic steric maps $\left(\% \mathrm{~V}_{\text {free }}, \% \mathrm{~V}_{\text {free }}{ }^{\text {quadr. }}\right)$ through $\mathrm{DFT}$ optimized structures and the Morfeus software (buried volumes and solvent accessible surface area and volumes). The third one is electronics-based descriptors calculated using DFT (HOMOLUMO-gaps, dipole moment, as well as the NBO charge on the central Ru-atom). Similarly, esters have also been represented by three types of chemical descriptors: (a) graph-based descriptors using an autocorrelation function of depth $4,{ }^{24}$ (b) sterics-based descriptors (Sterimol parameters and solvent accessible area and volume), and (c) electronics-based descriptors (HOMO-LUMO gap, dipole moment, $\mathrm{C}=\mathrm{O}$-stretching frequency and intensity, and NMR chemical shifts). Solvents and the nature of bases have also been found to play important roles in the catalytic output for this type of reaction, and therefore we also represented them with relevant descriptors. Solvents were represented by the dielectric constants and Gutman donor numbers, whereas bases were represented by their $\mathrm{pK}$-values. Additionally, solvents and base were also represented by the onehot-encoding.

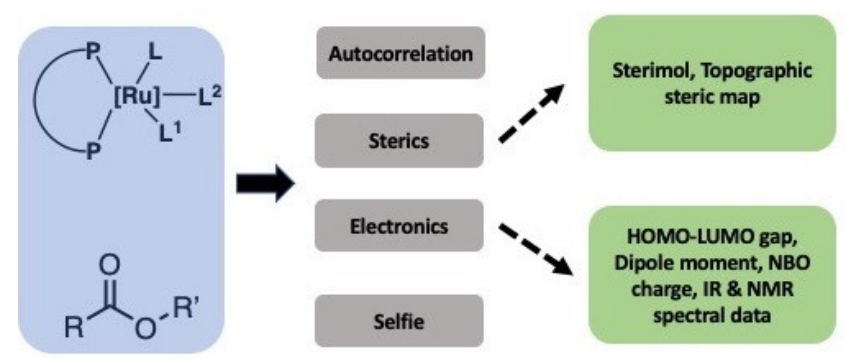

Figure 2. Summary of chemical descriptors for catalysts and esters used in this study.

\section{Development of a predictive model for the catalytic hy- drogenation of esters:}

3.1 Goals and description of ML architectures: In order to model the catalytic hydrogenation of esters using machine learning, we consider neural networks (NNs) and Gaussian processes (GPs). Our broader goals are two-fold: (1) predicting the yield of a hydrogenation reaction for a given set of reagents, catalyst, and reaction conditions, and (2) predicting catalysts that result in a high yield of hydrogenation reactions under mild conditions. As a first significant step towards the second goal, we $(2 \mathrm{a})$ predict the catalyst involved in a given chemical reaction from our compiled list of catalysts; and in a separate experiment (2b) predict the chemical descriptors of the catalysts. Therefore, we use a regression setting for (1) predicting reaction yields and ( $2 \mathrm{~b}$ ) predicting catalyst properties, whereas we use a classification setting for (2a). A detailed description of models and methodologies is provided in the SI. 
3.2 Prediction of yield: We started our investigations by the input of all the data points and the corresponding descriptors calculated using DFT, experiments (spectral data), and ML (one-hot-encoding) to the ML architectures. The dataset was divided into a training set and test set containing randomly selected $80 \%$, and $20 \%$ of the data respectively. The initial investigations showed that the mean accuracy for the prediction of yields was $78 \%$ (training set), and $74 \%$ (test set) using GP, whereas NN resulted in the mean accuracy of $79 \%$ (training set), and $75 \%$ (test set). Upon analyzing the plots of predicted yields vs true yields (see SI, Figure S2), we observed that the deviation of the low yielding data $(<50 \%)$ was significantly higher than those of the high yielding data $(>50 \%)$. We speculate this is due to a relatively low number of available data points for the reactions giving yields less than $50 \%$ (see Figure S1, SI). Furthermore, the available data points for the low yield reactions are not systematic, for example, a reaction could give low yield due to the use of a solvent or reagent that could poison the catalyst. This makes the ML architecture, which is primarily based on the quantity and quality of data, difficult to develop an accurate predictive model. Since our overall aim is to develop catalysts that could produce high yields and vice versa, we carried our ML studies on the data points of yields of more than $50 \%$. Remarkably, a mean accuracy of $98 \%$ for the training set and $91 \%$ for the test set was obtained using NN. A similar result was obtained using GP that resulted in the mean accuracy of $94 \%$ for training set and $91 \%$ for the test set (Figure 3). For NN, we performed a 5 -fold cross-validation on the training set over the hyperparameters of activation function, number of hidden layers, number of nodes in each layer, and dropout amounts (p). In case of GP, we carried leave-one-out cross-validation. Both the studies resulted in accuracies of $\sim 90 \%$ on the test set. More details on the estimation of errors of cross-validation for each run is provided in the SI (Section 3.2.1). Furthermore, using a linear regression, a slightly lower mean accuracy of $88 \%$ was obtained for the test set (yields $>50 \%$ ).
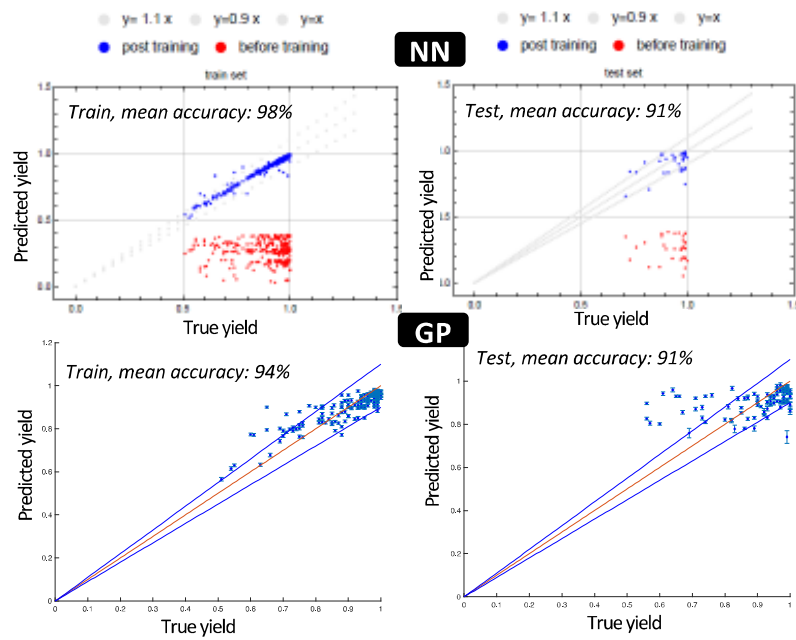

Figure 3. Prediction of yields for the catalytic hydrogenation of esters using NN and GP architectures for the dataset containing yield $>50 \%$.

It has been argued in the past that the choice of chemical descriptors plays a significant part in the robustness, and accuracy of the ML predictive model. ${ }^{21,32}$ To assess the significance of a particular type of chemical descriptors, we carried systematic
ML studies by choosing selective chemical descriptors. We divided a total of 20 descriptors into five categories: (a) autocorrelation function, (b) sterics of catalysts, characterized by free/buried volumes; (c) sterics of esters, characterized by free/buried volumes and Sterimol parameters; (d) electronics of catalysts, characterized by HOMO-LUMO gap, dipole moment, and NBO charge and (e) electronics of esters, characterized by dipole moment, HOMO-LUMO gap, and spectral parameters (NMR chemical shifts and IR stretching frequencies). 30 datasets were created using various combinations of these five types of chemical descriptors. Our experiments showed that the highest accuracy (e.g. $94 \%$ test set for the dataset of yield $>60 \%$ ) is obtained by just using electronics of catalysts and esters. Indeed, mechanistically electronics of both catalysts and esters play a significant role in the catalytic activity for the hydrogenation of esters. ${ }^{33-34}$ However, the accuracy is not significantly different from the case when some other selected descriptors are used. Interestingly, just using one-hot encoding without using a chemical descriptor, also resulted in the mean accuracy of $86 \%$ only slightly lower than our best result of $94 \%$ using selected descriptors. The results obtained using one-hot encoding were found to be very similar to those when all the descriptors were used. The studies were conducted using the dataset of different sizes containing data points of yields higher than a certain percentage (e.g. $50 \%, 60 \%$, etc ranging from 30 to $60 \%$ ) as depicted in Figure 4. Remarkably, in all the cases most accurate predictions were obtained using selected descriptors (electronics parameters of catalysts, and esters). These results suggest that valuable and interpretable information can be obtained from the ML models on factors governing high yield reaction outcomes and using relevant descriptors.

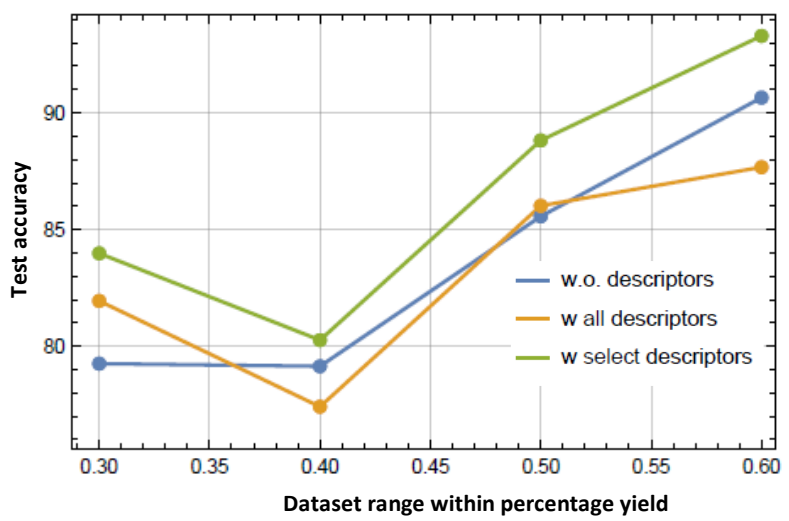

Figure 4. Plot of the accuracy of yield predictions relative to the size of the dataset (containing datapoints of yields higher than the given point).

3.3 Prediction of catalyst: Having developed an ML model for the prediction of yields, we diverted our attention to develop a model for the inverse problem - i.e. to predict a catalyst structure for the desired yield. To simplify the problem and demonstrate a proof of concept of using the ML approach for catalyst prediction, we turned this to a multi-channel classification problem asking our model to predict a particular catalyst given the reaction conditions and yields from the dataset. Gratifyingly, our model using the $\mathrm{NN}$ architecture predicted the corresponding catalysts (one-hot-encoding) with a mean accuracy of $85 \%$ (Figure 5). 


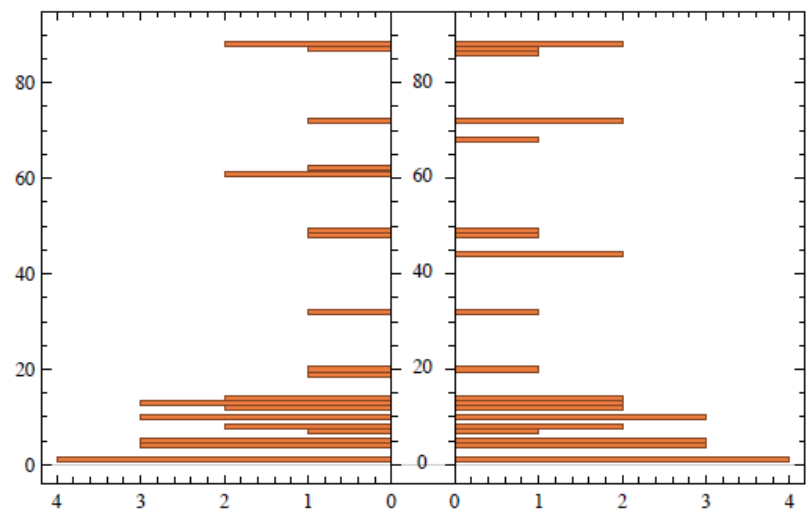

Figure 5. Histogram of actual vs predicted catalysts.

We employed a simple MLP (multilayer perceptron) architecture and linear regression to predict the various catalyst descriptors. In total we have 8 steric descriptors, and three electronic descriptors. Since neural networks are good at making end-to-end predictions, we attempted to predict all these features simultaneously. We conducted two different sets of experiments. In the first set of experiments [Expt. A], we divided our dataset into a train-test split of 80-20 and built an MLP model that aims to predict all such catalyst descriptors simultaneously. We compared our outcomes against linear models (realized through linear regression) for each of these features. In the second set of experiments [Expt. B], we divided the dataset into two disjoint parts such that each catalyst features in exactly one of the sets. This is in line with our goal of predicting new catalysts or their properties.

We state at the outset that our initial efforts in this direction have not yielded in predictions of high accuracy. This is largely in part due to the small amount of data available to us. We discovered that our models are good at predicting certain steric and electronic properties of catalysts. These are buried volume, solvent-accessible surface area and volume (SASA). Similarly, both the linear and the MLP models do quite well in predicting the HOMO-LUMO gap of the catalysts $(>90 \%$ test accuracy, see Table S5 in the SI). In Figures 6-7 we present a plot of true vs predicted HOMO-LUMO gaps and Buried volumes of catalysts from our models, showing a strong agreement. The data were standardized to keep the values of HOMO-LUMO gaps and Buried volumes in the range of $0-1$.

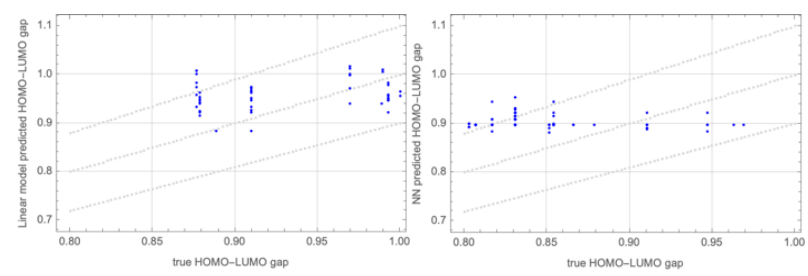

Figure 6. Prediction of HOMO-LUMO gap of Ru-catalysts by a (left) linear model and a (right) multi-layer perceptron model. This experimental set up corresponds to Expt. B detailed in Section 3.2 (SI) for cut-off yield $\gamma=0.5$. The dashed line in the middle is the line $\mathrm{x}=\mathrm{y}$. The other dashed lines reflect a $10 \%$ margin.
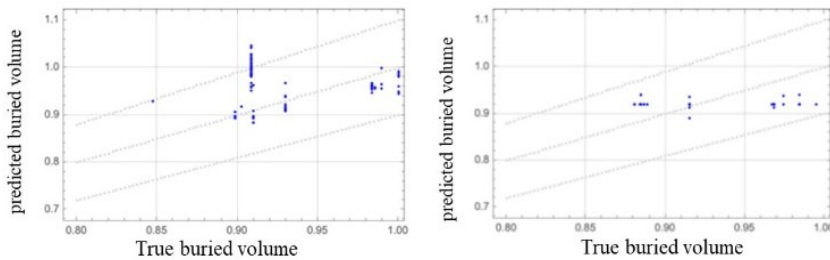

Figure 7. Prediction of buried volume of Ru-catalysts by a (left) linear model and a (right) multi-layer perceptron model. This experimental set up corresponds to Expt. B detailed in Section 3.2 (SI) for cut-off yield $\gamma=0.5$. The dashed line in the middle is the line $\mathrm{x}=\mathrm{y}$. The other dashed lines reflect a $10 \%$ margin.

In conclusion, we have demonstrated the approach of ML for the prediction of yields and corresponding catalyst descriptors for the hydrogenation of esters catalysed by well-defined ruthenium complexes. Our models can predict the reaction yields, and catalyst properties with high accuracies of up to $91 \%$, and $85 \%$, respectively. Our studies also indicate that the use of certain selected chemical descriptors (e.g. electronics parameters) in the model can outperform the prediction accuracies obtained using several other descriptors or just one-hot-encoding although these differences are in the range of 3-6\%. Moreover, we can also predict certain chemical descriptors with mean accuracies $>90 \%$. We note that the small size of the dataset with skewed population density (e.g. low datapoints for low yields, see Figure S1) is a limitation to our study which is primarily due to (a) difficulty in generating a large dataset of high quality by conducting high throughput/automation experiments that would need sophisticated equipment for a high pressure/temperature conditions, and (b) the limited availability of data (in particular of low yields) in the literature. We therefore would like to encourage the community to report low yielding results with their main discoveries and hope to develop a more generalizable model for the prediction of catalysts using a larger dataset in the future.

\section{ASSOCIATED CONTENT}

\section{Supporting Information}

The Supporting Information containing construction of the dataset, the creation of chemical descriptors, and the development of ML models is available on the ChemRxiv website. Code and data used in these analyses can be made available by authors on request. All computed structures are available at the ioChem-BD online repository under the following link: https://doi.org/10.19061/iochem-bd6-118.

\section{AUTHOR INFORMATION}

\section{Corresponding Author}

* Challenger Mishra: cm2099@cam.ac.uk

* Niklas von Wolff: niklas.von-wolff@u-paris.fr

* Amit Kumar: ak336@st-andrews.ac.uk

\section{Author Contributions}

$\mathrm{AK}, \mathrm{NvW}$, and $\mathrm{CM}$ prepared the manuscript. $\mathrm{AK}$ and $\mathrm{AP}$ created the dataset. NvW, EB, AK, and AP generated the chemical descriptors. CM, and AT developed the ML models. All authors have given approval to the final version of the manuscript.

\section{ACKNOWLEDGMENT}

We sincerely thank Prof. Neil Lawrence (Department of Computer Science and Technology, University of Cambridge) for his valuable 
advice and feedback on this project. AK thanks the Leverhulme Trust for an early career fellowship (ECF-2019-161). NVW and EB thank the IdEx Université de Paris (ANR-18-IDEX-0001) for funding. CM is supported by a Fellowship by the Accelerate Program for Scientific Discovery at the Computer Laboratory, University of Cambridge. The authors acknowledge the GENCI-CINES center for HPC resources (Projects A0080810359 and A0100810359).

\section{Notes}

The authors declare no competing financial interests.

\section{REFERENCES}

(1) Werkmeister, S.; Junge, K.; Beller, M. Catalytic Hydrogenation of Carboxylic Acid Esters, Amides, and Nitriles with Homogeneous Catalysts. Org. Process Res. Dev. 2014, 18 (2), 289-302.

(2) Kumar, A.; Gao, C. Homogeneous (De)Hydrogenative Catalysis for Circular Chemistry - Using Waste as a Resource. ChemCatChem 2021, 13 (4), 1105-1134.

(3) Clarke, M. L. Recent Developments in the Homogeneous Hydrogenation of Carboxylic Acid Esters. Catal. Sci. Technol. 2012, 2 (12), 2418-2423.

(4) Toyao, T.; Maeno, Z.; Takakusagi, S.; Kamachi, T.; Takigawa, I.; Shimizu, K. I. Machine Learning for Catalysis Informatics: Recent Applications and Prospects. ACS Catalysis. American Chemical Society February 7, 2020, pp 2260-2297.

(5) Kozlowski, M. C.; Dixon, S. L.; Panda, M.; Lauri, G. Quantum Mechanical Models Correlating Structure with Selectivity: Predicting the Enantioselectivity of $\beta$-Amino Alcohol Catalysts in Aldehyde Alkylation. J. Am. Chem. Soc. 2003, 125 (22), 6614-6615.

(6) Werth, J.; Sigman, M. S. Connecting and Analyzing Enantioselective Bifunctional Hydrogen Bond Donor Catalysis Using Data Science Tools. J. Am. Chem. Soc. 2020, 142 (38), 16382-16391.

(7) Tang, T.; Sandford, C.; Minteer, S. D.; Sigman, M. S. Analyzing Mechanisms in Co(i) Redox Catalysis Using a Pattern Recognition Platform. Chem. Sci. 2021, 12 (13), 4771-4778.

(8) Reid, J. P.; Sigman, M. S. Holistic Prediction of Enantioselectivity in Asymmetric Catalysis. Nature 2019, 571 (7765), 343-348.

(9) Sigman, M. S.; Harper, K. C.; Bess, E. N.; Milo, A. The Development of Multidimensional Analysis Tools for Asymmetric Catalysis and Beyond. Acc. Chem. Res. 2016, 49 (6), 1292-1301.

(10) Park, Y.; Niemeyer, Z. L.; Yu, J.-Q.; Sigman, M. S. Quantifying Structural Effects of Amino Acid Ligands in Pd(II)-Catalyzed Enantioselective C-H Functionalization Reactions. Organometallics 2018, 37 (2), 203-210.

(11) Werth, J.; Sigman, M. S. Linear Regression Model Development for Analysis of Asymmetric CopperBisoxazoline Catalysis. ACS Catal. 2021, 11 (7), 39163922.
Harper, K. C.; Bess, E. N.; Sigman, M. S. Multidimensional Steric Parameters in the Analysis of Asymmetric Catalytic Reactions. Nat. Chem. 2012, 4 (5), 366-374.

(13) Harper, K. C.; Sigman, M. S. Predicting and Optimizing Asymmetric Catalyst Performance Using the Principles of Experimental Design and Steric Parameters. Proc. Natl. Acad. Sci. 2011, 108 (6), 2179 LP - 2183.

Harper, K. C.; Sigman, M. S. Three-Dimensional Correlation of Steric and Electronic Free Energy Relationships Guides Asymmetric Propargylation. Science 2011, 333 (6051), 1875 LP - 1878.

(15) Sigman, M. S.; Harper, K. C.; Bess, E. N.; Milo, A. The Development of Multidimensional Analysis Tools for Asymmetric Catalysis and Beyond. Acc. Chem. Res. 2016, 49 (6), 1292-1301.

(16) Ahneman, D. T.; Estrada, J. G.; Lin, S.; Dreher, S. D.; Doyle, A. G. ORGANIC CHEMISTRY Predicting Reaction Performance in $C-N$ Cross-Coupling Using Machine Learning.

Zahrt, A. F.; Henle, J. J.; Rose, B. T.; Wang, Y.; Darrow, W. T.; Denmark, S. E. Prediction of Higher-Selectivity Catalysts by Computer-Driven Workflow and Machine Learning. Science 2019, 363 (6424).

(18) Singh, S.; Pareek, M.; Changotra, A.; Banerjee, S.; Bhaskararao, B.; Balamurugan, P.; Sunoj, R. B. A Unified Machine-Learning Protocol for Asymmetric Catalysis as a Proof of Concept Demonstration Using Asymmetric Hydrogenation. Proc. Natl. Acad. Sci. 2020, 117 (3), 1339 LP - 1345 .

(19) Beker, W.; Gajewska, E. P.; Badowski, T.; Grzybowski, B. A. Prediction of Major Regio-, Site-, and Diastereoisomers in Diels-Alder Reactions by Using Machine-Learning: The Importance of Physically Meaningful Descriptors. Angew. Chem. Int. Ed. 2019, 58 (14), 4515-4519.

(20) Durand, D. J.; Fey, N. Building a Toolbox for the Analysis and Prediction of Ligand and Catalyst Effects in Organometallic Catalysis. Acc. Chem. Res. 2021, 54 (4), 837-848.

(21) Durand, D. J.; Fey, N. Computational Ligand Descriptors for Catalyst Design. Chem. Rev. 2019, 119 (11), 65616594.

(22) Fey, N. Lost in Chemical Space? Maps to Support Organometallic Catalysis. Chem. Cent. J. 2015, 9 (1), 38.

Jover, J.; Fey, N.; Harvey, J. N.; Lloyd-Jones, G. C.; Orpen, A. G.; Owen-Smith, G. J. J.; Murray, P.; Hose, D. R. J.; Osborne, R.; Purdie, M. Expansion of the Ligand Knowledge Base for Chelating P,P-Donor Ligands (LKBPP). Organometallics 2012, 31 (15), 5302-5306.

(24) Friederich, P.; dos Passos Gomes, G.; De Bin, R.; AspuruGuzik, A.; Balcells, D. Machine Learning Dihydrogen Activation in the Chemical Space Surrounding Vaska's Complex. Chem. Sci. 2020, 11 (18), 4584-4601.
Jorner, K.; Brinck, T.; Norrby, P.-O.; Buttar, D. Machine 
Learning Meets Mechanistic Modelling for Accurate Prediction of Experimental Activation Energies. Chem. Sci. 2021, 12 (3), 1163-1175.

(26) Sinha, V.; Govindarajan, N.; de Bruin, B.; Meijer, E. J. How Solvent Affects $\mathrm{C}-\mathrm{H}$ Activation and Hydrogen Production Pathways in Homogeneous Ru-Catalyzed Methanol Dehydrogenation Reactions. ACS Catal. 2018, $8(8), 6908-6913$.

(27) Govindarajan, N.; Sinha, V.; Trincado, M.; Grützmacher, H.; Meijer, E. J.; de Bruin, B. An In-Depth Mechanistic Study of Ru-Catalysed Aqueous Methanol Dehydrogenation and Prospects for Future Catalyst Design. ChemCatChem 2020, 12 (9), 2610-2621.

(28) Zou, Y.-Q.; von Wolff, N.; Rauch, M.; Feller, M.; Zhou, Q.-Q.; Anaby, A.; Diskin-Posner, Y.; Shimon, L. J. W.; Avram, L.; Ben-David, Y.; Milstein, D. Homogeneous Reforming of Aqueous Ethylene Glycol to Glycolic Acid and Pure Hydrogen Catalyzed by Pincer-Ruthenium Complexes Capable of Metal-Ligand Cooperation. Chem. - A Eur. J. 2021, 27 (14), 4715-4722.

(29) Dub, P. A.; Gordon, J. C. Metal-Ligand Bifunctional Catalysis: The "Accepted" Mechanism, the Issue of Concertedness, and the Function of the Ligand in Catalytic Cycles Involving Hydrogen Atoms. ACS Catal. 2017, 7 (10), 6635-6655.

(30) Dub, P. A.; Gordon, J. C. The Mechanism of Enantioselective Ketone Reduction with Noyori and Noyori-Ikariya Bifunctional Catalysts. Dalt. Trans. 2016, 45 (16), 6756-6781.

(31) Kozuch, S.; Shaik, S. How to Conceptualize Catalytic Cycles? The Energetic Span Model. Acc. Chem. Res. 2011, 44 (2), 101-110.
(32) Estrada, J. G.; Ahneman, D. T.; Sheridan, R. P.; Dreher, S. D.; Doyle, A. G. Response to Comment on "Predicting Reaction Performance in C-N Cross-Coupling Using Machine Learning". Science 2018, 362, eaat8763.

(33) Sluijter, S. N.; Korstanje, T. J.; van der Vlugt, J. I.; Elsevier, C. J. Mechanistic Insights into Catalytic Carboxylic Ester Hydrogenation with Cooperative $\mathrm{Ru}(\mathrm{II})$-Bis $\{1,2,3-$ Triazolylidene\}Pyridine Pincer Complexes. J. Organomet.Chem. 2017, 845, 30- 37.

(34) Werkmeister, S.; Junge, K.; Beller, M. Catalytic Hydrogenation of Carboxylic Acid Esters, Amides, and Nitriles with Homogeneous Catalysts. Org. Process Res. Dev. 2014, 18, 289- 302. 


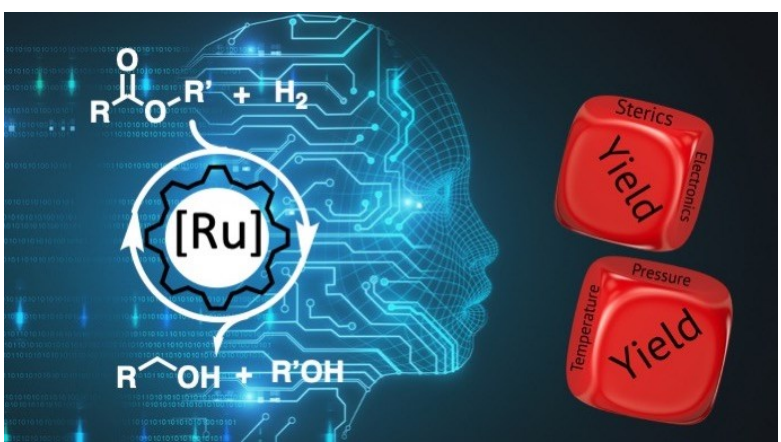

